\title{
Silicon surface modification with a mixed silanes layer to immobilize proteins for biosensor with imaging ellipsometry
}

\author{
Zhan-Hui Wang, Gang Jin* \\ National Microgravity Laboratory, Institute of Mechanics, Chinese Academy of Sciences, 15, Bei-si-huan West Road, Beijing 100080, PR China
}

Received 22 September 2003; received in revised form 2 December 2003; accepted 16 December 2003

\begin{abstract}
One kind of surface modification method on silicon wafer was presented in this paper. A mixed silanes layer was used to modify silicon surface and rendered the surface medium hydrophobic. The mixed silanes layer contained two kinds of compounds, aminopropyltriethoxysilane (APTES) and methyltriethoxysilane (MTES). A few of APTES molecules in the layer was used to immobilize covalently human immunoglobulin $\mathrm{G}(\mathrm{IgG})$ on the silicon surface. The human IgG molecules immobilized covalently on the modified surface could retain their structures well and bind more antibody molecules than that on silicon surface modified with only APTES. This kind of surface modification method effectively improved the sensitivity of the biosensor with imaging ellipsometry.
\end{abstract}

(c) 2004 Elsevier B.V. All rights reserved.

Keywords: Imaging ellipsometry; Mixed silanes layer; Biosensor

\section{Introduction}

The concept of biosensor based on imaging ellipsometry was reported several years ago [1]. Imaging ellipsometry is an enhancement of standard single-beam ellipsometry which combines the power of ellipsometry with microscopy [2]. A high spatial resolution in the order of micron (laterally) and sub-nanometer (vertically) can be achieved in bio-affinity-based sensing by imaging ellipsometry [1]. The field-of-view of the biosensor is so large (several square centimeters) that it is easy to detect multiple analytes on separated spots simultaneously and the surface concentration of protein on spots can be quantified without any labeling.

Silicon has been and is still widely used as the substrate in ellipsometric measurements due to the fact that its surface energy, polarity and surface chemistry can be easily controlled by silanization [3]. The hydrophobic silicon surface modified with dichlordimethylsilane is often used to adsorb proteins [4], but it involves some limitation. The adsorbed proteins suffer partial denaturation and tend to leach or wash off the surface [5,6]. The silicon surface was modified with 3-aminopropyltriethoxysilane (APTES), then the

\footnotetext{
* Corresponding author. Tel.: +86-10-62631816; fax: +86-10-62631816

E-mail address: gajin@imech.ac.cn (G. Jin).
}

amine group of APTES was reacted with glutaraldehyde to yield aldehyde group that could form an imine linkage with the primary amine group on proteins. This protein covalent immobilization method could make the proteins stable on the surface, but it did not yet overcome the problem of protein partial denaturation. It is reasonable to suppose that proteins adsorbed onto solid surfaces may undergo some conformational change because of the relatively low structural stability of proteins and their tendency to unfold to allow formation of additional contacts with the surface [7]. The degree of biological activity retention varies with the degree of protein native structure maintenance [8]. Surface properties have an enormous effect on the protein conformational change. Perhaps the most widely accepted generalization regarding surface properties concerns hydrophobicity and holds that the more hydrophobic the surface, the greater the change of protein conformation. Electrical charge is another general surface property that affects protein conformational change by electrostatic interactions. If silicon surface is modified to minimize these effects on protein conformational change, the proteins covalently immobilized on the silicon surface will retain native or near native structure and maintain high biological activity.

In this paper, a mixed silanes layer was used to modify silicon surface for the covalent immobilization of proteins. The mixed silanes layer contained two kinds of compounds, 
APTES and MTES. MTES was a nonionic and hydrophobic organic molecule. Medium hydrophobic silicon surface with little electrical charge would be obtained by the surface modification with the mixed silanes layer and the effect on protein conformational change caused by electrostatic interaction would be minimized. The hydrophobic interaction between surface and protein could be minimized by adding Tween 20 into the protein solution. Tween 20 is a non-ionic detergent. Some authors have reported that Tween 20 has a renaturating effect on antigens, resulting in improved recognition by specific antibodies [9]. In this paper, the human IgG covalently immobilized on the mixed silanes layer could bind more its antibody molecules than that on the silicon surface modified with only APTES, which indicated that the IgG molecule on the mixed silanes layer could retain its native structure better.

\section{Materials and methods}

\subsection{Chemicals and materials}

Chemicals used for the buffer (PBS, pH 7.4) preparation were all of analytical grade or better. APTES and MTES were purchased from Acros Organics (Belgium). Glutaraldehyde (50\% aqueous solution), Tween 20 and ethanolamine were purchased from Sigma-Aldrich. Polished silicon wafers were purchased from General Research Institute for Nonferrous Metals (China). Water was obtained from a Millipore milli-Q ion exchange apparatus. Human IgG and goat anti-IgG serum were obtained from Sigma (USA).

\subsection{Cleaning of silicon wafers}

The silicon wafers were cut into $5 \mathrm{~mm} \times 20 \mathrm{~mm}$ pieces and cleaned with a mixture of $30 \%$ hydrogen peroxide $\left(\mathrm{H}_{2} \mathrm{O}_{2}\right)$ and concentrated sulfuric acid $\left(\mathrm{H}_{2} \mathrm{SO}_{4}\right)(1: 3 \mathrm{v} / \mathrm{v})$ for $30 \mathrm{~min}$. After thoroughly rinsed with water and pure ethanol, the slides were stored in pure ethanol before use.

\subsection{Silanization of cleaned silicon wafer surfaces with APTES}

The cleaned wafers were reacted with a fresh ethanol solution of APTES (5\% APTES, 5\% water and 90\% pure ethanol) for $1 \mathrm{~h}$ at room temperature, followed by the rinsing with water three times and pure ethanol three times, and then stored in pure ethanol. This procedure rendered the surface medium hydrophobic, with the water advancing contact angle of $50^{\circ} \pm 1^{\circ}$ and receding contact angle of $45^{\circ} \pm 0.8^{\circ}$.

\subsection{Silanization of cleaned silicon wafer surfaces with MTES}

The cleaned slides were reacted with a fresh ethanol solution of MTES (5\% MTES, 5\% water and 90\% pure ethanol)
[10] for $1 \mathrm{~h}$ at room temperature, followed by rinsing with water three times and pure ethanol three times, and then stored in pure ethanol. This procedure rendered the surface medium hydrophobic, with a water advancing contact angle of $58^{\circ} \pm 1^{\circ}$ and receding contact angle of $54^{\circ} \pm 0.5^{\circ}$.

\subsection{Silanization of cleaned silicon wafer surfaces with APTES and MTES}

The cleaned wafers were reacted with a fresh ethanol solution of APTES and MTES (1:30, mol/mol, $5 \%$ water and $90 \%$ pure ethanol) for $1 \mathrm{~h}$ at room temperature, followed by rinsing with water three times and pure ethanol three times, and then stored in pure ethanol. This procedure rendered the surface medium hydrophobic, with a water advancing contact angle of $55^{\circ} \pm 1.2^{\circ}$ and receding contact angle of $47^{\circ} \pm 0.5^{\circ}$.

\subsection{Covalent immobilization of human $\operatorname{Ig} G$}

The silicon surfaces modified with APTES and mixed silanes layer were reacted with a $2.5 \%$ solution of glutaraldehyde in PBS buffer for $2 \mathrm{~h}$, followed by rinsing with PBS buffer. The glutaraldehyde surfaces were then placed into $0.1 \mathrm{mg} / \mathrm{ml}$ human $\mathrm{IgG}$ solution with the addition of $1 \%$ Tween 20 at room temperature until the saturated human IgG layers were obtained. The surfaces were washed with PBS buffer and the remaining aldehyde groups on the surfaces were deactivated with $1 \mathrm{M}$ ethanolamine for $30 \mathrm{~min}$.

\subsection{Human $\operatorname{Ig} G$ binding with anti-IgG}

The silicon surfaces coupled with human IgG were placed into $0.1 \mathrm{mg} / \mathrm{ml}$ anti-IgG solution with the addition of $1 \%$ Tween 20 and allowed to incubate for $30 \mathrm{~min}$ at room temperature, followed by rinsing with water and dried with nitrogen.

\subsection{Biosensor with imaging ellipsometry}

The experiments were carried out with a biosensor based on imaging ellipsometry developed in our laboratory. Imaging ellipsometry was an enhancement of standard single-beam ellipsometry, which combined the power of ellipsometry with microscopy and worked in the off-null mode. The imaging ellipsometry used in this study was an automated one and ellipsometric conditions could be controlled by auto-adjusting the polariser and the analyzer; the angle of incidence was variable from $45^{\circ}$ to $90^{\circ}$ with a resolution of $0.05^{\circ}$; the magnification of image to object is modulated according to the dimension of the field of view, so that the lateral resolution of $3 \mu \mathrm{m}$ could be reached; auto-focusing was realized with the standard of the Laplacian algorithm. All the adjustments were carried out automatically with micro-stepping motors controlled by a computer with home-made software. With the same 
software, the digital images in grayscale format ( 8 bits, 0-255 grayscale) could be also automatically captured and processed. The light source was a Xenon lamp, and a specific collimating system was used to provide an expanded parallel probe beam with a diameter of about $25 \mathrm{~mm}$. The beam passed through a polarizer and a compensator (a quarter wave plate) and finally onto the sample at an incident angle of $75^{\circ}$. An optical filter at $633 \mathrm{~nm}$ wavelength was placed in the incident optical passage to select wavelength in order to increase the ellipsometric contrast of image. The reflection beam passed through an analyzer and an imaging lens with a spatial filter located at its focus plane, and then the ellipsometric image was focused onto the sensing area of the CCD camera. For a sample with lateral distribution of layer thickness (or surface concentration), null ellipsometry could not be carried out over the entire surface simultaneously due to the fact that different areas would yield different polarization changes. In order to overcome the problem, the optical components in the immunosensor were adjusted to fulfill the null conditions on a silicon wafer surface without adsorbed layers and the off-null ellipsometric principle was used to measure the adsorption layer thickness distribution (or surface concentration). Under this condition, the detected intensity " $\Gamma$ " was related to the thickness $(d)$ of the layer according to $I=k d^{2}$ [2]. As for the same protein and the same ellipsometric conditions, $k$ is a constant and can be determined by the protein layer with known intensity in grayscale and its absolute thickness. In this paper, several samples of protein adsorption layer were prepared and the intensity in grayscale and the absolute thickness were measured with imaging ellipsometry and conventional ellipsometer, respectively. The value of the constant $\mathrm{k}$ was then calculated, with which the absolute thickness of other protein layer was calculated directly from the intensity measured by imaging ellipsometry. For protein adsorption layer measured in air, the protein adsorption amount could be calculated according to the relationship between surface concentration and protein layer thickness, surface concentration $\left(\mathrm{ng} / \mathrm{mm}^{2}\right) \approx K \times d(\mathrm{~nm})$, where $K \approx 1.2$ [11].

\section{Ellipsometer}

The thickness of biomolecular layers was calibrated with an ellipsometer of rotating analyzer type (SE 400, SENTECH, Germany) equipped with a He-Ne laser $(\lambda=$ $632.8 \mathrm{~nm})$.

\section{Results and discussion}

\subsection{Surface modification}

The silicon surface used in this paper was modified with three methods. One of the methods was using APTES to silanize silicon surface, yielding amido groups with positive charges on surface (named APTES surface). Another was using MTES to modify surface. MTES was a nonionic organic molecule and the surface modified with it was medium hydrophobic (named MTES surface). The third was using APTES and MTES (1:30, mol/mol) to modify silicon surface. The modified surface was also medium hydrophobic with the positive charges yielded between the first two surfaces (named APTES/MTES surface). These three kinds of modified silicon surfaces had different surface properties. In order to determine how these different surface properties affected protein adsorption, the following experiment was carried out. The three kinds of surfaces were partly inserted into $0.1 \mathrm{mg} / \mathrm{ml}$ human IgG solution with the addition of $1 \%$ Tween 20 for $30 \mathrm{~min}$, respectively. After rinsed with water and dried with nitrogen, these surfaces were detected with imaging ellipsometry and the digital images of these surfaces in grayscale format ( 8 bits, 0-255 grayscale) were recorded with a computer. The results were shown in Fig. 1. A human $\operatorname{IgG}$ layer with about $5.0 \pm 0.5 \mathrm{~nm}$ was formed on the APTES surface (Fig. 1A) and about $1.2 \pm 0.3 \mathrm{~nm}$ on APTES/MTES surface (Fig. 1B), but no human IgG was adsorbed on the MTES (Fig. 1C). The result indicated that Tween 20 could inhibit hydrophobic interaction that cause proteins physical adsorption on solid surface and prevent protein from adsorbing on the MTES surface. The electrostatic interaction caused by a few positive charges on the APTES/MTES surface may be the reason for the adsorption of a little human IgG. The physical adsorption of human IgG on the APTES surface may be the same consequence of the electrostatic interaction. The images in the grayscale format were converted into three-dimensional images according to the relationship between the intensity and the thickness of the layer and shown in Fig. 1D-F.

\subsection{Covalent immobilization of human $\operatorname{Ig} G$}

In order to keep human IgG stable on silicon surface, covalent immobilization is the preferred method. APTES and APTES/MTES surfaces could be used to immobilize human IgG covalently, because the amido groups on these two surfaces could react with Glutaraldehyde (Glu), yielding aldehyde groups that could form an imine linkage with the primary amine groups in the exposed lysine residues on the surface of protein. To compare with these two kinds of surfaces' capacity of protein immobilization, the surfaces were activated with Glutaraldehyde, and then partly incubated into human $\operatorname{IgG}$ solution with the addition of $1 \%$ Tween 20 until the saturated human IgG layers formed. The result was shown in Fig. 2. The amount of human IgG immobilized covalently on APTES-Glu surface was almost same as that on APTES/MTES-Glu surface. The thickness of the saturated human IgG layer was $5.0 \pm 0.5 \mathrm{~nm}$. The molecular weight of human IgG is $150 \mathrm{kDa}$, while APTES is only 221 . The size of IgG is also much larger than that of APTES. Using the dimensions of IgG molecules $(4.5 \mathrm{~nm} \times 4.5 \mathrm{~nm} \times 23.5 \mathrm{~nm}$ [12]), the theoretical adsorbed amounts at complete surface coverage for vertical and hor- 


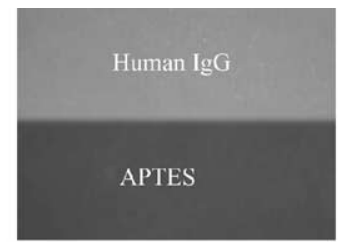

(A)

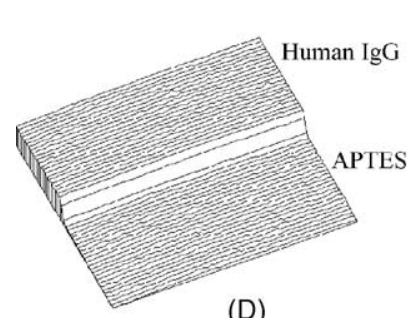

(D)

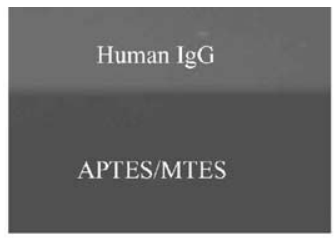

(B)

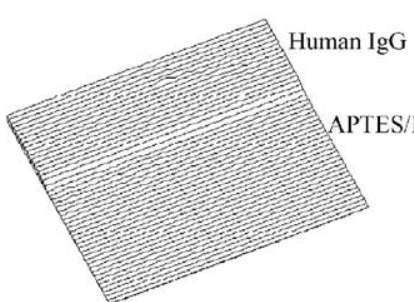

(E)

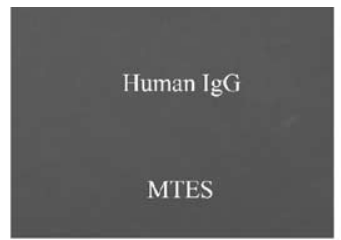

(C)

Fig. 1. The physical adsorption of human IgG on APTES, APTES/MTES and MTES surfaces with the addition of $1 \%$ Tween 20 in $0.1 \mathrm{mg} / \mathrm{ml}$ human IgG solution. A human IgG layer with the thickness of about $5.0 \pm 0.5 \mathrm{~nm}$ was formed on the APTES surface (A), about $1.2 \pm 0.3 \mathrm{~nm}$ on APTES/MTES surface (B), and no human IgG was adsorbed on the MTES(C); D, E and F were images in 3-D converted from the video pictures of A, B and C, respectively.
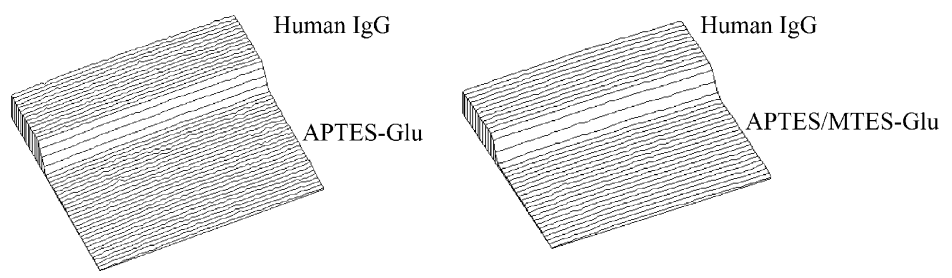

Fig. 2. Human IgG covalently immobilized on APTES-Glu and APTES/MTES-Glu surfaces. The amount of human IgG covalently immobilized on these two kinds of surfaces was almost the same and the thickness of human IgG layer was about $5.0 \pm 0.5 \mathrm{~nm}$.

izontally oriented adsorption are 12.02 and $2.30 \mathrm{ng} / \mathrm{mm}^{2}$, respectively [13]. According to the relationship between surface concentration and protein layer thickness, surface concentration $\left(\mathrm{ng} / \mathrm{mm}^{2}\right) \approx K \times d(\mathrm{~nm})$, where $K \approx 1.2$ [11], the surface concentration of human IgG on both APTES-Glu and APTES/MTES-Glu was about $6 \mathrm{ng} / \mathrm{mm}^{2}$. The result indicated that the adsorption amount of human IgG was between those of complete surface coverage for vertical and horizontally oriented adsorption. The amount of APTES on the APTES/MTES surface was sufficient for the immobilization of almost a completely covered layer of human IgG. In fact, one imine linkage may be enough for one IgG molecule covalent immobilization on surface. More bonds formed between $\operatorname{IgG}$ molecule and surface would make the molecule become more rigid and result in partial denaturation [14].

\subsection{Antibodies binding capacity}

The maintenance of functional configuration of protein molecules immobilized on solid surface was the key element in the development of biosensor with high sensitivity. In order to compare the biological activity of human IgG immobilized on APTES-Glu, APTES/MTES-Glu and APTES surfaces, these three kinds of surfaces coupling with human $\mathrm{IgG}$ were incubated into human $\mathrm{IgG}$ polyclonal antibodies (anti-IgG) solution with same concentration for the same period of time. The results were shown in Fig. 3. The highest amount of bound anti-IgG was found on the APTES/MTES-Glu surface $(11 \pm 0.7 \mathrm{~nm})$ followed by APTES-Glu $(9.5 \pm 0.5 \mathrm{~nm})$ and APTES $(7.5 \pm 0.5 \mathrm{~nm})$ surfaces. The data indicated that the surface property clearly

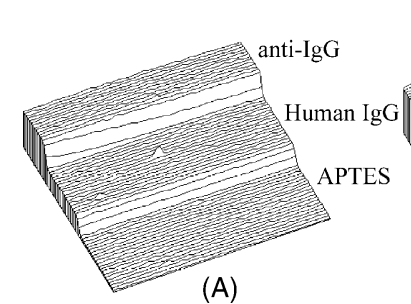

(A)

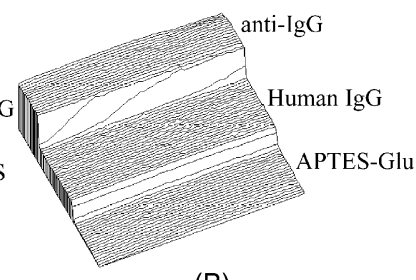

(B)

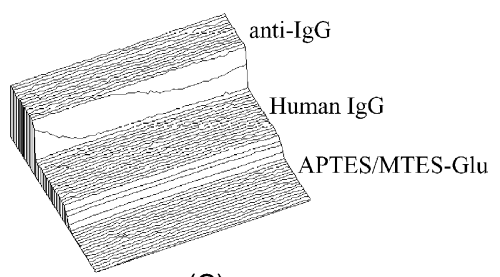

(C)

Fig. 3. Human IgG immobilized on APTES, APTES-Glu and APTES/MTES-Glu surfaces bound with its antibody. The amount of human IgG immobilized covalently on these three kinds of surfaces were almost the same and the thickness of human IgG layer was about $5.0 \mathrm{~nm}$. The highest amount of bound anti-IgG was found on the APTES/MTES-Glu surface $(11 \pm 0.7 \mathrm{~nm})$ followed by APTES-Glu $(9.5 \pm 0.5 \mathrm{~nm})$ and APTES $(7.5 \pm 0.5 \mathrm{~nm})$ surfaces. 
affected the binding between human IgG and its antibody. The human IgG on APTES/MTES-Glu surface may maintain more native sites for anti-IgG to bind. More bonds formed between human $\mathrm{IgG}$ molecule and surface may result in partial denaturation of human IgG on APTES-Glu surface. On the APTES surface, the electrostatic interactions may be the main reason for the partial denaturation of human $\mathrm{IgG}$.

\section{Conclusions}

One mixed silanes layer contained two kinds of compounds, APTES and MTES, was used to modify silicon surface for human IgG immobilization in this paper. The physical adsorption of human IgG on the mixed silanes layer was less than that on the surface modified with APTES. The human IgG molecules immobilized covalently on APTES/MTES-Glu surface could retain their structures better and bind more antibody molecules than that on APTES-Glu surface. The result was shown here only for a demonstration purpose. In a real application, the appropriate ratio of APTES to MTES should be determined according to relevant protein immobilized and the proper immobilization conditions should be optimized.

The results in this study showed that surface modification method with mixed silanes layer of APTES and MTES could be used to optimize the sensitivity of the biosensor with imaging ellipsometry, which has a potential for a sensitive immunoassay technique.

\section{Acknowledgements}

The national natural science foundation of China and Chinese Academy of Sciences are acknowledged for their supports.

\section{References}

[1] G. Jin, P. Tengvall, Anal. Biochem. 232 (1995) 69-72.

[2] G. Jin, R. Jansson, H. Arwin, Rev. Sci. Instrum. 67 (1996) 29302936.

[3] H. Arwin, Thin solid films 313-314 (1998) 744-764.

[4] U. Jonsson, B. Ivarsson, I. Lundstrom, L. Berghem, J. Colloid Interf. Sci. 90 (1982) 148.

[5] S.K. Bhatia, L.C. Shriver-Lake, K.J. Prior, J.H. Georger, J.M. Calvert, R. Bredehorst, F.S. Ligler, Anal. Biochem. 178 (2) (1989) 408413.

[6] L.C. Shriver-Lake, B. Donner, R. Edelstein, K. Breslin, S.K. Bhatia, F.S. Ligler, Biosens. Bioelectron. 12 (11) (1997) 1101-1106.

[7] T.A. Horbett, Cardiovasc. Pathol. 2 (1993) 137s-148s.

[8] R.K. Sandwick, K.J. Schray, J. Colloid Interf. Sci. 121 (1988) 112.

[9] A.P. Van Dam, H.G. Van den Brink, R.J.T. Smeenk, J. Immunol. Methods 129 (1990) 63-70.

[10] H. Yuan, W.M. Mullett, J. Pawliszyn, Analyst 126 (8) (2001) 14561461.

[11] M. Stenberg, H. Nygren, J. Phys. 44 (1983) 83-86.

[12] M. Malmsten, Colloids Surf. B 3 (1995) 297-308.

[13] S.E. Moulton, J.N. Barisci, A. Bath, R. Stella, G.G. Wallace, J. Colloid Interf. Sci. 261 (2003) 312-319.

[14] J.N. Lin, J. Herron, J.D. Andrade, M. Brizgys, IEEE Trans. Biomed. Eng. 35 (1988) 466-471. 